\title{
Primary intraosseous meningioma of the vertebra: illustrative case
}

\author{
Ue-Cheung Ho, MD, ${ }^{1}$ Koping Chang, MD, ${ }^{2}$ Yen-Heng Lin, MD, MS, ${ }^{3}$ Yu-Cheng Huang, MD, ${ }^{3}$ and Fon-Yih Tsuang, MD, PhD ${ }^{1}$ \\ ${ }^{1}$ Division of Neurosurgery, Department of Surgery, and Departments of ${ }^{2}$ Pathology and ${ }^{3}$ Medical Imaging, National Taiwan University Hospital, Taipei City, Taiwan
}

BACKGROUND Primary intraosseous meningiomas (PIMs) are rare, and PIMs of the vertebrae have not yet been reported. The authors report a case of primary meningioma arising from the vertebrae.

OBSERVATIONS A 49-year-old man presented with lower back pain and numbness in both lower extremities. Lumbar spine magnetic resonance imaging revealed an L2 pathological fracture with epidural and paraspinal invasion. The patient had undergone a first palliative decompression and fixation surgery, and the diagnosis turned out to be a World Health Organization grade III anaplastic meningioma based on histopathology. The tumor had progressed after first operation and radiation therapy, and the patient was referred to the authors' institute for excision. The patient had an uneventful postoperative course after a revisional total en bloc spondylectomy of $\mathrm{L} 2$.

LESSONS The authors present a rare case of PIM of the vertebrae with epidural and paraspinal invasion. Careful preoperative assessment and surgical planning is crucial for successful patient management.

https://thejns.org/doi/abs/10.3171/CASE21362

KEYWORDS primary intraosseous meningioma; primary extradural meningioma; anaplastic meningioma; primary spine tumor; total en bloc spondylectomy

Meningiomas are the most common primary intracranial tumors and comprise approximately $35 \%$ of all primary central nervous system tumors. ${ }^{1,2}$ Most meningiomas develop intracranially. In rare cases, they can develop extracranially; however, such cases account for less than $2 \%$ of all meningiomas. ${ }^{3-5}$ This type of meningioma is called primary extradural meningioma (PEM), and it can occur in several locations, such as the skull, skin, paranasal sinuses, orbit, temporal fossa, and oral cavity. ${ }^{3,4,6,7}$ Meningiomas can also metastasize to skull, bone, or spine from a primary intracranial origin, ${ }^{2,3,8,9}$ or they can develop as a primary intraosseous meningioma (PIM) in bone. PIMs are a subset of PEMs and are defined as meningiomas having the largest tumor contained within the calvaria and having no or only minimal dural involvement. ${ }^{10-12}$ To date, none of the literature has reported on cases of intraosseous meningiomas present in only the vertebrae without evidence of metastasis from other sites. We report a rare case of a PIM located at the L2 vertebrae, and the lesion was consistent with a
World Health Organization (WHO) grade III anaplastic meningioma based on the histopathology results.

\section{Illustrative Case}

A 49-year-old man, without a medical history of cancer or trauma, presented with low back pain and numbness in both lower extremities starting 9 months prior to admission. On neurological examination, he had hypesthesia to light touch bilaterally along the L2-3 dermatomes. His muscle power was a 5 of 5 in all extremities, and his muscle stretch reflexes were normoreflexic bilaterally. Deep anal pressure was present. Spine magnetic resonance imaging (MRI) performed at another facility revealed a pathological fracture of $L 2$, with the $L 2$ vertebral body mass measuring $5.3 \mathrm{~cm}$ in diameter, bilateral psoas muscle invasion, and tumor extension into the epidural space between the lower of $L 1$ to upper $L 3$. The gadolinium-enhanced MRI sequence revealed a heterogeneous enhancement (Fig. 1). The tumor caused an indentation of the thecal sac

ABBREVIATIONS CT = computed tomography; MRI = magnetic resonance imaging; $\mathrm{PEM}=$ primary extradural meningioma; $\mathrm{PIM}=$ primary intraosseous meningioma; WHO = World Health Organization.

INCLUDE WHEN CITING Published October 18, 2021; DOI: 10.3171/CASE21362.

SUBMITTED June 16, 2021. ACCEPTED July 2, 2021.

(C) 2021 The authors, CC BY-NC-ND 4.0 (http://creativecommons.org/licenses/by-nc-nd/4.0/). 

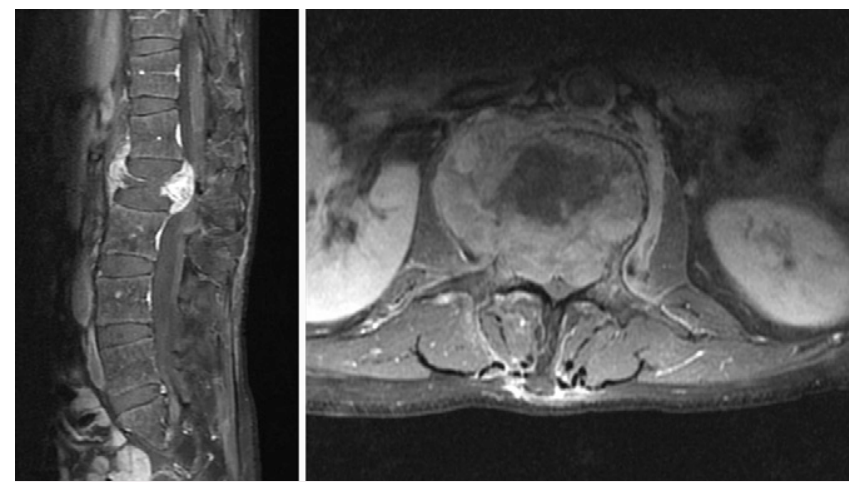

FIG. 1. Spine MRI before the first operation. Left: Gadoliniumenhanced T1-weighted sagittal image showed a pathological fracture of L2 with soft tissue mass $5.3 \mathrm{~cm}$ in diameter. Right: Gadoliniumenhanced T1-weighted axial image revealed L2 tumor with bilateral psoas muscle invasion and epidural extension.

with compression of the cauda equina. With a tentative diagnosis of an L2 metastasis, the patient had first undergone palliative decompression and posterior fixation surgery on November 4, 2020 at a different facility. Histopathology turned out to be an anaplastic meningioma. An MRI of the brain was obtained after the operation due to the concern for metastasis from the intracranial region; however, it did not reveal tumors in the brain or cranium. His lower back pain and numbness in the lower extremities resolved after the operation. Following first palliative decompression surgery, the patient received a total dose of $50 \mathrm{~Gy}$ in 25 fractions using volumetric modulated arc therapy delivered to the residual tumor over paraspinal region. However, his back pain and numbness in the both lower extremities returned in February 2021. The subsequent spinal $M R I$ at the end of February revealed an enlarged $L 2$ residual tumor with thecal sac compression and psoas muscle invasion (Fig. 2). He was referred to our hospital for excision. Due to the concern for the possibility of a diagnosis other than meningioma, which rarely presents as a primary tumor in the vertebrae, he had repeated computed tomography (CT)-guided biopsy. The pathology result
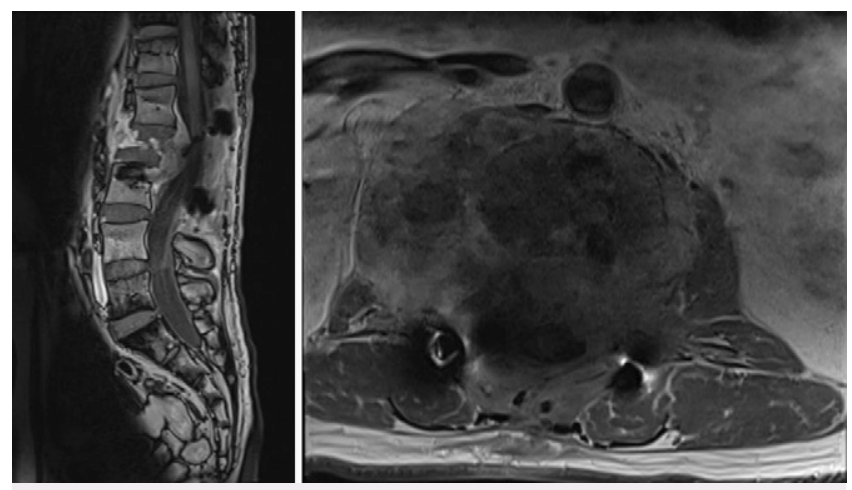

FIG. 2. Spine MRI 4 months after the first palliative decompression surgery. Left: Gadolinium-enhanced T1-weighted sagittal image revealed an enlarged residual tumor. Right: Gadolinium-enhanced T1-weighted axial image revealed an enlarged tumor with more extensive bilateral psoas muscle invasion. came back as meningioma. Lumbar spine dual-energy CT for tumor vascularity showed a major blood supply from the right L2 radicular artery with mild arteriovenous shunting inside the tumor. ${ }^{13} \mathrm{~A}$ wholebody positron emission tomography scan showed no other metastasis. Transarterial embolization had been performed 1 day before the scheduled operation. He received a revisional total en bloc spondylectomy of L2 via a combined posterior-anterior approach. The length of the operation was 505 minutes, and the total estimated blood loss was $2200 \mathrm{~mL}$. A postoperative lumbar radiographs and spinal MRI revealed complete removal of the tumor and adequate implant position (Figs. 3 and 4). The tumor was confirmed as a WHO grade III anaplastic meningioma based on histopathology, and the resected margin near the right psoas muscle was involved (Fig. 5). The patient had fair ambulation and less back pain and numbness in the bilateral lower extremities after the excision. He will undergo a total dose of $25 \mathrm{~Gy}$ in 5 fractions radiotherapy delivered to the right $\mathrm{L} 1-2$ paraspinal region due to the involved margin in the pathology report.

\section{Discussion}

\section{Observations}

In the past, the nomenclature for meningioma arising outside of the intracranial compartment varied across studies. Ectopic, heterotopic, extracalvarial, calvarial, or extracranial meningiomas are terms that have been used to describe meningiomas in these regions. ${ }^{4,7,14}$ Recently, the nomenclature "primary extradural meningioma" was used to describe primary meningiomas arising outside the intracranial compartment. ${ }^{4}$ PEMs can occur in multiple locations, such as in the skull, skin, paranasal sinuses, orbit, temporal fossa, and oral cavity and, in rare cases, it can occur in the lung, mediastinum, adrenal gland, and finger. ${ }^{3,4,6,7}$ It has been classified as purely extracalvarial (type I), purely calvarial (type II), or calvarial with extracalvarial extension (type III). ${ }^{4}$ PIM is defined as a
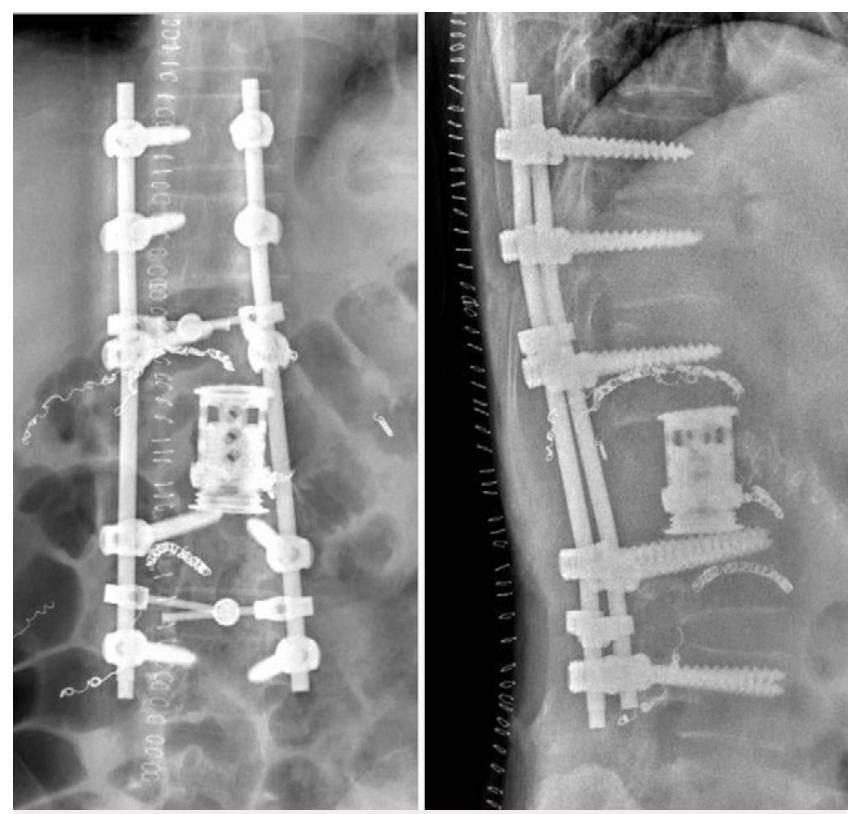

FIG. 3. Left and right: Spine radiographs after excision showed the instrumentation, reconstruction, and embolization coils. 

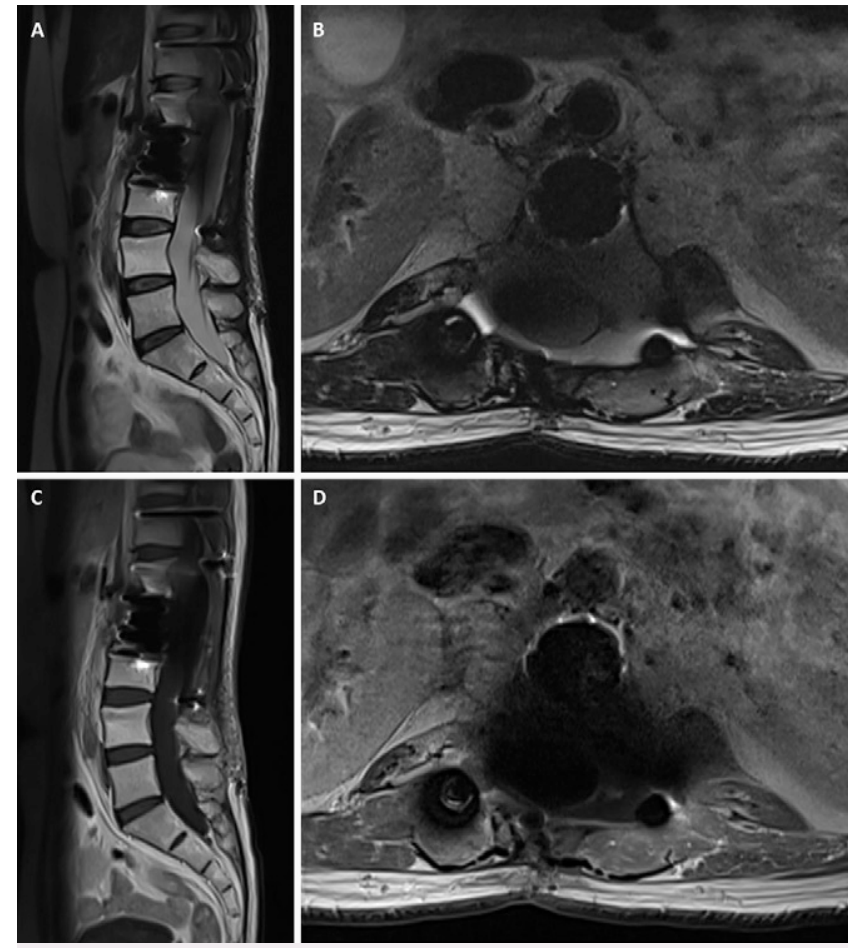

FIG. 4. Spine MRI 1 week after excision. Sagittal and axial T2-weighted images (A and $\mathbf{B}$ ) and sagittal and axial gadolinium-enhanced T1weighted images ( $C$ and $\mathbf{D}$ ) show no residual tumor radiologically.

meningioma, with the largest tumor contained within the calvaria with no or only minimal dural involvement. ${ }^{10-12}$ Although the original paper that proposed the classification system for PEM considered PIM a different entity from PEM based on the sex predilection, age distribution, and tendency for malignancy, some considered PIM as a subset of PEM based on the anatomical considerations. ${ }^{3,6,8,14-17}$ By definition, PIMs arise from the bone, particularly the calvaria, and the sphenoid bone and frontal bone are the most common locations, followed by the parietal bone, temporal bone, orbit, and other regions. ${ }^{3,11,12}$ There have been a few reports in recent decades of intraosseous meningiomas arising from the vertebrae where meningiomas had metastasized to the vertebrae from their intracranial origin; therefore, these tumors cannot be categorized into "primary" intraosseous meningiomas. ${ }^{2,3}$ To date, only 1 article has reported on a rare case of concurrent meningiomas arising in the lung and vertebra without any evidence of tumors present in the head and neck regions. ${ }^{8}$ The authors considered the lung as most likely the primary origin of the meningioma rather than the vertebral bone because no cases of PIM outside the head and neck region had been previously reported. Herein, we present a rare case of PIM of the vertebra, which has not been described previously.

The majority of PIMs are of osteoblastic origin and have the typical findings of hyperostosis; however, some have radiographic evidence of osteolytic changes. $3,6,12,14,17$ The differential diagnosis of osteolytic lesions in vertebrae includes metastasis, primary bone tumors, multiple myeloma, Langerhans cell histiocytosis, or osteomyelitis. Biopsy is indicated to differentiate between the osteolytic lesions because most primary bone tumors necessitate wide excisions
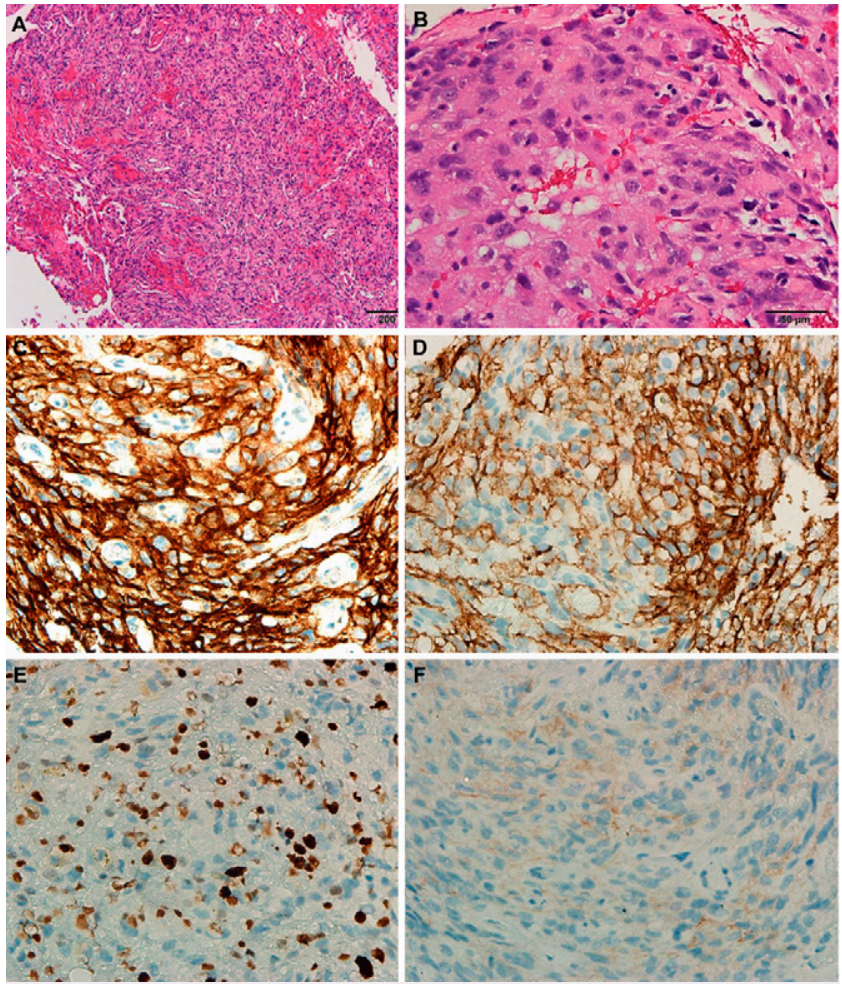

FIG. 5. A and B: Hematoxylin and eosin staining of tumor showed some foci of spindle tumor cells with significant nuclear pleomorphism, increased nucleocytoplasmic ratio, and prominent nucleoli. C and D: Tumor cells stained positive for SSTR2a and E-cadherin. E: The Ki-67 cell proliferation index was approximately $15-20 \%$. F: Tumor cells focally stained positive for D2-40. Original magnifications $\times 100(\mathbf{A})$ and $\times 400(B-F)$.

to achieve better clinical outcomes. The case presented here had palliative decompression and fixation surgery at another hospital and had rapid progression of the tumor after that operation. This highlights the importance of appropriate surgical management. We performed a second biopsy on the lesion to confirm the diagnosis and then arranged an imaging study to confirm that the vertebra was the primary site of the disease. We then performed surgery, and the patient had a tumor resection with wide surgical margins in accordance with standard oncological principles to achieve Enneking-appropriate margin; otherwise, we called the surgery Enneking-inappropriate. ${ }^{18}$ The final pathology report came back as a WHO grade III anaplastic meningioma. This is compatible with a previous study showing that PEMs are prone to the development of malignancy. ${ }^{3,4,6,14-16,19}$ Thus, maximum tumor resections that can be done safely and adjuvant radiotherapy are necessary in the management of these patients.

There are several proposed mechanisms for the development of PEM, including the presence of arachnoid cap cells trapped in the cranial sutures during birth, fractures that develop after traumatic events, or the development from multipotent mesenchymal cells that are capable of meningothelial differentiation., ${ }^{4,15}$ The latter could explain the development of intraosseous meningiomas located far from the head and neck and could explain how vertebrae could become primary sites of intraosseous meningioma. 


\section{Lessons}

The importance of adequate preoperative evaluation and appropriate surgical management for those patients with uncertain nature of spine lesion cannot be overemphasized. Every solitary spine tumor in patients without other extraspinal lesions should first be managed as a primary malignant bone tumor. Preoperative biopsy and detailed imaging study are needed to differentiate primary spine lesion from metastatic lesion. Oncological principle with en bloc resection is mandatory in the management of a patient with primary spine lesion. We performed revisional excision after an Ennekinginappropriate surgery for a rare case of PIM at the spine. The pathologic diagnosis was a WHO grade III anaplastic meningioma. Careful preoperative assessment and surgical planning is crucial for successful patient management, especially for those whose diagnosis of primary bone tumors is suspected.

\section{References}

1. Buerki RA, Horbinski CM, Kruser T, Horowitz PM, James CD, Lukas RV. An overview of meningiomas. Future Oncol. 2018;14(21):2161-2177.

2. Ward AL, Risman A, Segar S, Sharma S, Vender JR. Atypical intracranial meningioma with metastasis to $\mathrm{C} 7$ vertebral body: a case report. World Neurosurg. 2019;122:593-598.

3. Kim H, Jung TY, Kim IY, Lee JK. Two cases of primary osteolytic intraosseous meningioma of the skull metastasizing to whole skull and the spine. J Korean Neurosurg Soc. 2012;51(3):151-154.

4. Lang FF, Macdonald OK, Fuller GN, DeMonte F. Primary extradural meningiomas: a report on nine cases and review of the literature from the era of computerized tomography scanning. J Neurosurg. 2000;93(6):940-950.

5. Elder JB, Atkinson R, Zee CS, Chen TC. Primary intraosseous meningioma. Neurosurg Focus. 2007;23(4):E13.

6. Kwon SM, Ko Y, Bang SS. Primary intraosseous osteolytic meningioma: a case report and review of the literature. BMC Neurol. 2019;19(1):176

7. Rushing EJ, Bouffard JP, McCall S, et al. Primary extracranial meningiomas: an analysis of 146 cases. Head Neck Pathol. 2009;3(2):116-130.

8. Tamura $\mathrm{H}$, Otani $\mathrm{Y}$, Iwazawa $\mathrm{T}$, et al. Extracranial meningiomas concurrently found in the lung and vertebral bone: a case report. $J$ Med Case Reports. 2018;12(1):279.

9. Mukherjee D, Hu JL, Chu RM. Isolated extracranial intraosseous metastasis of an intracranial meningioma following bevacizumab therapy: case report and review of the literature. Asian J Neurosurg. 2018;13(1):98-101.

10. Arana $\mathrm{E}$, Diaz $\mathrm{C}$, Latorre FF, et al. Primary intraosseous meningiomas. Acta Radiol. 1996;37(6):937-942.

11. Butscheidt S, Ernst M, Rolvien T, et al. Primary intraosseous meningioma: clinical, histological, and differential diagnostic aspects. $J$ Neurosurg. 2020;133(2):281-290.

12. Omofoye OA, Huynh T, Jhun R, Ashfaque H, Cronk K. Primary intraosseous meningioma of the calvarium: a systematic review. Clin Neurol Neurosurg. 2020;199:106283.

13. Huang YC, Tsuang FY, Lee CW, Wu CY, Lin YH. Assessing vascularity of osseous spinal metastases with dual-energy CT-DSA: a pilot study compared with catheter angiography. AJNR Am J Neuroradiol. 2019;40(5):920-925.

14. Chen TC. Primary intraosseous meningioma. Neurosurg Clin N Am. 2016;27(2):189-193.

15. Choi KW, Chung KJ, Kim YH. Primary intraosseous meningioma Arch Plast Surg. 2015;42(3):378-380.

16. Vlychou M, Inagaki Y, Stacey R, Athanasou NA. Primary intraosseous meningioma: an osteosclerotic bone tumour mimicking malignancy. Clin Sarcoma Res. 2016;6:14.

17. Caruso R, Fini G, Pesce A, et al. A primary intraosseous cystic meningioma: case report. Int J Surg Case Rep. 2017;37:189-192.

18. Boriani S. En bloc resection in the spine: a procedure of surgical oncology. J Spine Surg. 2018;4(3):668-676.

19. Harary M, Tung JK, Sood S, Corrales CE, Smith T, lorgulescu JB. Benign purely intraosseous meningioma of the skull: diagnosis and surgical outcomes. J Clin Neurosci. 2020;82(Pt A):36-42.

\section{Disclosures}

The authors report no conflict of interest concerning the materials or methods used in this study or the findings specified in this paper.

\section{Author Contributions}

Conception and design: Tsuang, Ho. Acquisition of data: all authors. Analysis and interpretation of data: Ho, Chang. Drafting the article: Tsuang, Ho. Critically revising the article: Tsuang. Reviewed submitted version of manuscript: Tsuang. Approved the final version of the manuscript on behalf of all authors: Tsuang. Administrative/technical/ material support: Huang.

\section{Correspondence}

Fon-Yih Tsuang: National Taiwan University Hospital, Taipei City, Taiwan. tsuangfy@gmail.com. 